\title{
ORIGINAL
}

\section{RIESGO CARDIOVASCULAR DEL PACIENTE HIPERTENSO CON SEGUIMIENTO PROLONGADO EN ATENCIÓN PRIMARIA. EL EFECTO DEL ENVEJECIMIENTO (CICLO RISK STUDY) (*)}

\author{
Luis García-Ortiz (1), Manuel A. Gómez-Marcos(1), Luis J. González-Elena (2), José A \\ Maderuelo-Fernández (2), Emilio Ramos-Delgado (1) y Miguel Torrecilla-Garcia (3).
}

(1) Unidad de Investigación La Alamedilla. Centro de Salud La Alamedilla. Salamanca.

(2) Gerencia de Atención Primaria de Salamanca.

(3) Centro de Salud de San Juan. Salamanca.

\section{RESUMEN}

Fundamento La evolución del riesgo cardiovascular estimado, puede servir para valorar la efectividad de las diferentes intervenciones terapéuticas que se realizan en pacientes con seguimiento habitual en las consultas del médico de familia. El objetivo de este trabajo es comparar diferentes sistemas de evaluación del efecto de las intervenciones preventivas en la evolución del riesgo coronario en el seguimiento a largo plazo de personas hipertensas en Atención Primaria.

Métodos: Estudio descriptivo longitudinal con seguimiento de 8.42 pacientes hipertensos de 34 a 70 años durante 6 años en dos centros de atención primaria, con una intervención de mejora de calidad (ciclo de mejora) en el último año en uno de ellos. El seguimiento mínimo en atención primaria previo al inicio del estudio fue de dos años. Las variables principales fueron edad y sexo, presión arterial, lípidos, tabaquismo, diabetes y riesgo cardiovascular (RCV)(Framingham-Wilson) en la situación real y considerando constante la edad en el primer supuesto y los factores de riesgo en el segundo y el riesgo relativo.

Resultados: Se encontró un descenso de la presión arterial sistólica y diastólica de $11,78 \mathrm{mmHg}$ (IC95\%:10,51-13,05) y $8,83 \mathrm{mmHg}$ (IC95\%:8,13-9,53) respectivamente y LDL-Colesterol 15,94 mg/dl (IC95\%:11,77-20,12), un ascenso del HDLColesterol de 7,53 mg/dl (IC95:6,39-8,66), disminución del tabaquismo del $31 \%$ y un aumento de diabéticos. El riego coronario disminuyó 1,40(IC95\%:0,87-1,93) puntos porcentuales, el RCV con edad constante descendió 3,84(IC95\%:3,35-4,33) y con factores de riesgo constantes incrementó 3,06(IC95\%:2,823,29). El Riesgo relativo descendió de 2,50 a 1,85.

Conclusiones: El envejecimiento puede enmascarar el efecto logrado por la atención sanitaria en el control del riesgo cardiovascular absoluto. El riesgo relativo podría ser una alternativa para monitorizar el seguimiento.

Palabras clave: Hipertensión. Riesgo coronario. Envejecimiento. Atención primaria de salud.

Correspondencia:

Luís García Ortiz

Centro de Salud La Alamedilla. Unidad de Investigación

Av. Comuneros 27-31. Ciudad: Salamanca CP: 37003

Correo electrónico: Lgarciao@usal.es

\section{ABSTRACT}

\section{Cardiovascular Risk of Hypertensive People with Long-Range Monitoring. The Effect of Aging (Ciclo Risk Study)}

Background: The evolution of estimated cardiovascular risk can be for evaluating the effectiveness of the different treatment interventions which are carried out on patients with regular follow-up by family physicians. This study is aimed at evaluating the effect of aging on the evolution of cardiovascular risk among hypertensive patients with long-range Primary Care monitoring.

Methods: Longitudinal, descriptive study with monitoring of 842 hypertensive patients within the 34-70 age range at two primary care centers, with a quality improvement intervention (improvement cycle) during the last year at one of these centers. The main variables were age and gender, blood pressure, lipids, smoking habit, diabetes and cardiovascular risk (CVR) (Framingham-Wilson) in the real-life situation and considering the age constant in the first case, and the risk factors in the second, plus the relative risk.

Results: A drop was found in the systolic and diastolic blood pressure from $11.78 \mathrm{mmHg}$ (95 IC: 10.51-13.05) and $8.83 \mathrm{mmHg}$ (95 CI: 8.13-9.53), respectively, and LDL Cholesterol $15.94 \mathrm{mg} / \mathrm{dl}$ (95 CI: 11.77-20.12), a rise in HDL-Cholesterol of $7.53 \mathrm{mg} / \mathrm{dl}$ (95CI: 6.39-8.66), decreased smoking habit of $31 \%$ and an increase in diabetics. The Coronary risk decreased 1.40 percent points, Coronary risk with age constant decreased 3.84 (95 CI: 3.35-4.33), having increased with constant risk factors by 3.06(95 CI: 2.82-3.29). The Relative risk dropped from 2.50 to 1.85 .

Conclusions: Aging may mask the effect achieved by health care in the absolute cardiovascular risk check. The relative risk could be an alternative for monitoring the follow-up.

Key words: Hypertension. Cardiovascular risk. Aging. Primary health care.

(*) Financiación: Este trabajo ha sido financiado por el ISCIII-RETCI RD06/0018 y la Consejería de Sanidad de la Junta de Castilla y León. 


\section{INTRODUCCIÓN}

El riesgo cardiovascular absoluto ${ }^{1}$, entendido como la probabilidad de sufrir un evento cardiovascular en un tiempo determinado, es una herramienta recomendada por las guías clínicas actuales ${ }^{2-7}$ y cada vez más utilizado por los clínicos en el abordaje terapéutico de los distintos factores de riesgo cardiovascular.

Dentro del concepto de riesgo cardiovascular se incluye la probabilidad de padecer las enfermedades arterioscleróticas más importantes: cardiopatía isquémica, enfermedad cerebrovascular y arteriopatía periférica. Sin embargo, existen múltiples escalas que utilizan diferentes metodologías y diferentes variables para la estimación del riesgo cardiovascular, como es el riesgo de morbimortalidad coronaria (Framingham y Regicor) ${ }^{8-11}$, riesgo de mortalidad cardio y cerebrovascular $(\mathrm{SCORE})^{12}$, riesgo de morbi-mortalidad cardio y cerebrovascular (European Society Hypertension $)^{7}$, así como escalas especificas para diabéticos (UKPDSRisk) ${ }^{13}$. Los riesgos estimados por las diferentes escalas no siempre coinciden y pueden originar cierta confusión y dificultar su aplicabilidad practica, como se ha podido observar en diferentes estu$\operatorname{dios}^{14-21}$.

La evolución en el tiempo del riesgo cardiovascular estimado puede servir para valorar la efectividad de las diferentes intervenciones terapéuticas que se realizan en personas con seguimiento habitual en las consultas de atención primaria. Sin embargo, su envejecimiento progresivo, junto con el aumento del riesgo cardiovascular que conlleva ${ }^{22-23}$ por el importante peso que tiene la edad en todas las escalas de riesgo, puede estar enmascarando el efecto real de las intervenciones realizadas ${ }^{24-25}$. Por esta razón, habitualmente, en el seguimiento a largo plazo que se realiza en el ámbito de la atención primaria de pacientes crónicos, es difícil conseguir mantener la reducción inicial alcanzada con las intervenciones terapéuticas realizadas.

El riesgo relativo $(\mathrm{RR})$ o razón respecto al bajo riesgo, entendido como la razón del riesgo coronario absoluto de cada sujeto y el riesgo de un sujeto de la misma edad y sexo con riesgo coronario bajo ${ }^{26}$, podría ser un instrumento útil para analizar la evolución del riesgo a medio y largo plazo, al ser independiente de la edad y del sexo de los pacientes. No obstante, las indicaciones terapéuticas basadas en los grandes ensayos clínicos se han realizado en función del riesgo cardiovascular absoluto.

Aunque existen numerosas publicaciones en las que se estima el riesgo cardiovascular con diferentes escalas y metodologías, en nuestro medio no hemos encontrado trabajos en los que se valorara la evolución en el tiempo del riesgo coronario absoluto y relativo estimado y la influencia que puede tener el paso del tiempo en la modificación de dicha estimación.

El estudio ciclo-risk ${ }^{19,27-30}$ se diseñó para valorar la efectividad de intervenciones de mejora de calidad (ciclos de mejora) en relación al descenso del riesgo cardiovascular en pacientes hipertensos.

El objetivo de este artículo es comparar diferentes sistemas de evaluación del efecto de las intervenciones preventivas en la evolución del riesgo coronario en el seguimiento a largo plazo de pacientes hipertensos en atención primaria

\section{SUJETOS Y MÉTODOS}

Se realizó un estudio con un diseño descriptivo ambispectivo con una intervención de mejora de calidad sobre los profesionales sanitarios en el último año, en dos centros de atención primaria. Los dos cen- 
tros están ubicados en el mismo edificio, tienen una plantilla mayoritariamente estable desde 1990, son docentes pre y pos grado y tienen unas características y metodología de trabajo similares. Los protocolos de control y seguimiento del paciente hipertenso han sido similares en ambos centros, así como las sesiones de formación continuada que se desarrollan habitualmente en conjunto, salvo las específicas de la intervención de calidad antes reseñada.

La primera fase fue un estudio observacional, descriptivo y retrospectivo, con la información recogida de los pacientes hipertensos seleccionados, desde que iniciaron su control o se diagnosticaron de hipertensión arterial en los centros de salud participantes, anualmente y hasta el momento del comienzo del estudio o evaluación pre-intervención, valorando los factores de riesgo y el riesgo coronario con la escala de Framingham (Wilson 1998) ${ }^{9}$. Posteriormente se realizó una intervención de mejora de calidad mediante la metodología de ciclos de mejora, consistente en auditoria de historias clínicas (audit), feedback y sesiones para la implementación de guías clínicas en uno de los centros de salud, sirviendo los profesionales del otro como grupo de control, lo cual fue motivo de otra publicación ${ }^{27}$. Finalmente se realizó una nueva evaluación un año después.

Se seleccionó a todos los pacientes hipertensos entre 35 años y 70 años, ya que el rango de edad de la escala de Framingham es de 35 a 74 y se preveían varios años de seguimiento, diagnosticados de hipertensión arterial y con seguimiento habitual en los centros de salud. Se excluyó a los que seguían el control de la hipertensión en otro dispositivo asistencial y los que tenían un seguimiento inferior a dos años en los centros de salud al inicio del estudio. En total se incluyeron 842 hipertensos, de los cuales eran mujeres
535 (63,5\%). El cálculo del tamaño muestral se estimó para detectar una diferencia de una unidad porcentual en el riesgo coronario, aceptando un riesgo alfa de 0,05 y un riesgo beta de 0,20 para un contraste bilateral con una desviación estándar de 5,7 y una estimación de perdidas de seguimiento del $1 \%$. Se precisaban en total 802 sujetos, por lo que se consideró la inclusión de todas las personas hipertensas que cumplían los criterios descritos.

La información ha sido obtenida a través de la historia clínica por cuatro evaluadores entrenados previamente. Las variables medidas han sido la edad, sexo, presión arterial sistólica (PAS) y diastólica (PAD), lípidos (HDL y LDL-Colesterol), tabaquismo, diabetes, riesgo coronario utilizando la escala de Framingham (Wilson 1998) ${ }^{9}$ y estimación de riesgo relativo coronario (RR) entendido como la razón entre el riesgo coronario absoluto de cada paciente y el riesgo de un paciente de la misma edad y sexo con riesgo coronario bajo ${ }^{26}$. Se consideró bajo riesgo de enfermedad coronaria cuando el individuo no era diabético ni fumador, la presión arterial sistólica estaba por debajo de $120 \mathrm{mmHg}$ y la diastólica de $80 \mathrm{mmHg}$, el colesterol total estaba entre 160 y 199 $\mathrm{mg} / \mathrm{dl}$ o el colesterol unido a lipoproteínas de baja densidad (cLDL) entre 100 y 129 $\mathrm{mg} / \mathrm{dl}$ y el de alta densidad (cHDL) era superior a $45 \mathrm{mg} / \mathrm{dl}$ en varones y $55 \mathrm{mg} / \mathrm{dl}$ en mujeres ${ }^{9-26 .}$

Al ser un estudio descriptivo longitudinal y pretender evaluar únicamente la evolución del riesgo coronario con diferentes metodologías y no la efectividad de una intervención, el análisis de los datos en este trabajo se realizó del conjunto de personas hipertensas, independientemente del grupo en el que se incluyó en el estudio de mejora de calidad.

Se plantearon tres escenarios en la valoración de la evolución del riesgo coronario 
absoluto. En primer lugar el riesgo crudo o real calculado con la escala de Framingham-Wilson anualmente desde la fecha de inicio del seguimiento en los centros de salud. En segundo lugar, manteniendo constante la edad con la que se inició el seguimiento en los centros a la hora de la estimación del riesgo y modificando únicamente los factores de riesgo coronario según los resultados de la valoración anual, este escenario valoraría la efectividad de la intervención sanitaria en el descenso del riesgo cardiovascular estimado, aunque sin la influencia de la edad en la modificación del riesgo. No obstante no se puede valorar la influencia de la edad en la modificación de los factores de riesgo que intervienen en la ecuación del cálculo del riesgo. En tercer lugar, manteniendo constantes el nivel de los factores de riesgo que tenia el paciente cuando se inició su seguimiento y modificando solamente la edad en cada valoración, es decir la persona envejece pero los factores de riesgo no se modifican.
En el análisis estadístico, además de la estimación de medias y proporciones, se utilizaron el test de McNemar para el análisis de variables cualitativas apareadas y la $\mathrm{T}$ de Student para el análisis de variables cuantitativas independientes y apareadas. Para el contraste de hipótesis se fija un riesgo a de 0.05 . El paquete estadístico utilizado ha sido el SPSS/PC+ (V.14.0)

\section{RESULTADOS}

El seguimiento medio desde el inicio ha sido de 6,92 años, con una mediana de 6 años. Las pérdidas de seguimiento desde la valoración pre-intervención han sido 51 (17 varones y 34 mujeres), lo que supone un $6 \%$, sin que prácticamente se altere la estructura de sexo de la muestra y sin diferencia estadísticamente significativa en el riesgo coronario previo a la intervención entre ese grupo $(16,70 \%)$ y el resto $(15,62 \%)$. En la tabla 1 se observa la evolución de los factores de riesgo al inicio del

Tabla 1

Evolución de los factores de riesgo y riesgo cardiovascular desde la situación basal y la final

\begin{tabular}{|l|c|c|c|c|c|}
\hline \multicolumn{1}{|c|}{ Medición (n) } & Basal (842) & Año 1 (842) & Año2 (842) & Pre Int. (842) & Final (791) \\
\hline EDAD & $56,28(7,32)$ & $57,28(7,32)$ & $58,24(7,27)$ & $61,05(7,13)$ & $62,12(7,10)^{*}$ \\
\hline SEXO n $^{\mathbf{0}}$ mujeres $(\%)$ & $535(63,5)$ & $535(63,5)$ & $535(63,5)$ & $535(63,5)$ & $501(63,3)$ \\
\hline PAS (mmHg) & $153,47(16,79)$ & $143,98(14,28)$ & $143,20(13,66)$ & $142,74(14,57)$ & $141,53(13,85)^{*}$ \\
\hline PAD $(\mathbf{m m H g )}$ & $95,63(8,71)$ & $89,65(7,39)$ & $89,14(7,27)$ & $88,63(7,84)$ & $86,67(7,80)^{*}$ \\
\hline CT mg/dl & $228,33(42,61)$ & $226,32(38,94)$ & $225,25(38,75)$ & $230,93(39,91)$ & $224,88(36,86)^{*}$ \\
\hline LDL-C mg/dl & $169,22(40,61)$ & $167,35(37,15)$ & $162,62(37,50)$ & $160,06(38,60)$ & $148,35(33,70)^{*}$ \\
\hline HDL-C mg/dl & $48,07(11,84)$ & $48,22(11,86)$ & $49,08(12,58)$ & $53,93(15,10)$ & $55,80(15,15)^{*}$ \\
\hline DIABETES n $(\%)$ & $127(15,3)$ & $130(15,7)$ & $132(16,2)$ & $142(17,2)$ & $143(18,3)^{*}$ \\
\hline FUMADOR n $\%)$ & $122(15,0)$ & $108(13,3)$ & $99(12,4)$ & $86(10,6)$ & $79(10,4)^{*}$ \\
\hline RCV real & $16,46(9,70)$ & $15,23(8,94)$ & $15,40(9,27)$ & $15,69(9,26)$ & $14,95(9,30)^{*}$ \\
\hline RCV con edad constante & $16,46(9,70)$ & $14,67(8,64)$ & $14,35(8,76)$ & $13,60(8,37)$ & $12,50(7,99)^{*}$ \\
\hline RCV con FR constantes & $16,46(9,70)$ & $17,09(10,04)$ & $17,65(10,29)$ & $18,93(10,79)$ & $19,41(11,17)^{*}$ \\
\hline Riesgo Relativo & $2,50(1,23)$ & $2,20(1,07)$ & $2,15(1,04)$ & $2,02(0,98)$ & $1,83(0,91) *$ \\
\hline
\end{tabular}

Los valores presentados son media (desviación estándar), salvo los expresados en \%.

PAS : Presión arterial sistólica, PAD: presión arterial diastólica, CT Colesterol total, LDL-C: LDL-Colesterol, HDL-C: HDL-Colesterol, RCV :Riesgo cardiovascular. * p $<0.05$ entre evaluación basal y evaluación final. 
Figura 1

Evolución del riesgo coronario absoluto (RC) (Escala de Framingham-Wilson) desde la situación basal a la final

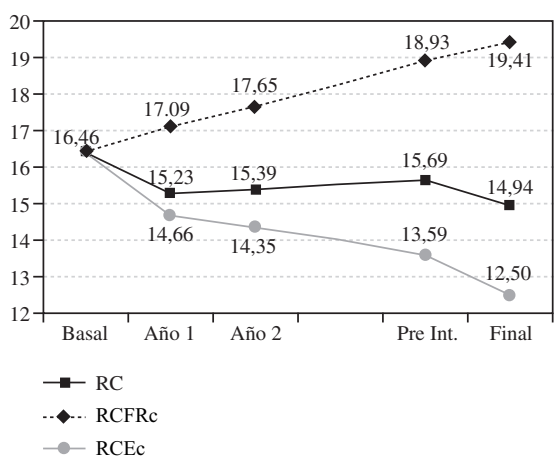

RC: Riesgo coronario real. RCEc: Riesgo coronario con la edad constante. RCFRc: Riesgo coronario con los factores de riesgo constantes. Basal: Medición inicio de seguimiento. Año 1: medición el primer año de seguimiento, Año 2: medición $2^{\circ}$ año de seguimiento. Pre int: Medición previa a la intervención de mejora de calidad. Post int: Medición al año de la intervención de mejora de calidad.

control en los centros de salud (medición basal) y en las mediciones sucesivas hasta la finalización del estudio.

El descenso medio de la PAS fue de $11,78 \mathrm{mmHg}$ (IC95\%:10,51-13,05) y la PAD $8,83 \mathrm{mmHg}$ (IC95\%:8,13-9,53). El LDL-Colesterol descendió 15,94 mg/dl (IC95\%:11,77-20,12) y el HDL-Colesterol subió 7,53 mg/dl (IC95\%:6,39-8,66). El porcentaje de fumadores también experimentó un descenso continuo $(31 \%$ de disminución desde la situación basal), mientras que el número de nuevos diabéticos sufrió un aumento progresivo del $15 \%$ al $18 \%$.

El riesgo coronario real estimado (tabla 1 y figura 1) disminuyó durante el primer año de control del $16,46 \%$ al $15,23 \%$ $(\mathrm{p}<0.001)$, es decir 1,23 puntos porcentuales(IC95\%:0,88 a 1,57) para luego iniciar un incremento progresivo sólo interrumpido por el efecto de la intervención de mejora de calidad que consiguió un descenso del riesgo del $15,69 \%$ al $14,95 \%$
Figura 2

Evolución del riesgo relativo coronario (RR) desde la situación basal a la final

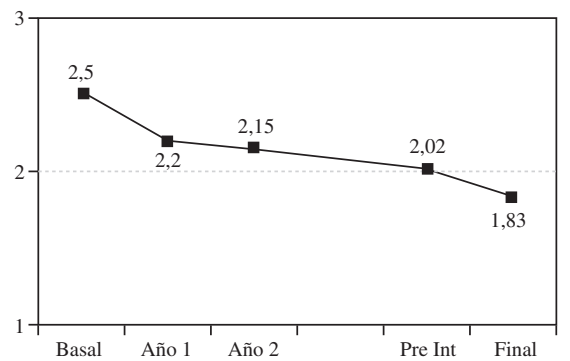

Basal: Medición inicio de seguimiento. Año 1: medición el primer año de seguimiento, Año 2: medición $2^{\circ}$ año de seguimiento. Pre int: Medición previa a la intervención de mejora de calidad. Final: Medición al año de la intervención de mejora de calidad.

$(\mathrm{p}=0.007)$. Por lo tanto, el descenso del riesgo coronario real desde la valoración basal a la final (no considerando las perdidas) fue de 1,40 (IC95\%:0,87-1,93) $(\mathrm{p}<0.05)$. Sin embargo, no se modifica prácticamente desde el riesgo alcanzado después del primer año de seguimiento $(15,23 \%)$ hasta la valoración final $(14,95 \%)(\mathrm{p}=0,45)$

En el escenario teórico con mantenimiento de la edad constante el descenso de riesgo coronario fue de 3,84 (IC95\%:3,354,33) $(\mathrm{p}<0.001)$. Por otro lado, en el escenario en el que los factores de riesgo permanecen constantes en el tiempo y el paciente continua envejeciendo, el riesgo coronario desde la situación basal experimentó un incremento constante, que en este caso fue de 3,06 (IC95\% 2,82$3,29)(\mathrm{p}<0.001)$.

Por ultimo, como se puede observar en la tabla 1 y figura 2 el riesgo relativo ha experimentado un descenso constante desde el inicio del seguimiento, con una medición basal de 2,50 hasta la valoración final (evaluación post intervención) de 1,83, es decir un descenso de 0,66 (IC95\%:0,59 a 0,74) puntos y si valoramos el la reducción desde 
el primer año $(2,20)$ hasta la final esta ha sido de 0,37 (IC95\%:0.30 a 0,43) puntos $(\mathrm{p}<0.001)$.

\section{DISCUSIÓN}

El importante descenso de los factores de riesgo coronario alcanzado en las personas hipertensas en la consulta de atención primaria parece no corresponderse con el mínimo descenso del riesgo coronario absoluto a largo plazo. Este hecho puede llevar a pensar en un aparente fracaso terapéutico si utilizamos únicamente la evolución del riesgo coronario absoluto estimado, como se viene recomendando en las guías clínicas ${ }^{2-7}$, para monitorizar la eficacia terapéutica de la atención habitual a las personas hipertensas. Como se muestra en la tabla 1 y la figura 1 , después de un importante descenso el primer año de seguimiento, el riesgo coronario absoluto permaneció prácticamente constante con tendencia al alza en el seguimiento a largo plazo. $\mathrm{El}$ descenso del riesgo coronario del primer año se explica por el inicio de las actuaciones sanitarias, bien por ser un nuevo diagnóstico de hipertensión arterial o por nueva adscripción al centro de salud y por lo tanto inclusión en el programa de control y seguimiento de la hipertensión arterial.

Este aparente fracaso terapéutico observado en nuestro estudio se puede explicar por la influencia del paso del tiempo, pues la edad es una variable importante en las escalas de riesgo coronario y al aumentar durante los primeros 4 años de seguimiento el nivel de riesgo se va elevando y amortigua los esfuerzos realizados para controlar los factores de riesgo cardiovascular modificables, puesto que la estructura de la muestra no se modifica durante el seguimiento salvo el incremento progresivo de la edad.

Teniendo en cuenta que contra el paso del tiempo no podemos intervenir, mante- ner la edad constante en la estimación del riesgo nos aproxima a la situación que observaríamos si el envejecimiento no estuviera incrementando el riesgo, sin tener en cuenta que también suele empeorar la situación de los otros factores de riesgo que intervienen en la ecuación.

Así tendríamos que frente a un descenso real del riesgo desde el inicio del seguimiento hasta la valoración final de 1,40 puntos porcentuales, el descenso teórico sería de 3,84. En el otro extremo estaría la evolución teórica del riesgo coronario si se mantuvieran constantes los factores de riesgo, pese a que la tendencia natural sería empeorar si no se interviene. El solo hecho del paso del tiempo hubiese elevado el riesgo coronario medio de estas personas hasta casi el $20 \%$ en la valoración al final del seguimiento, es decir, un ascenso mayor de 3 puntos porcentuales. Estos tres puntos del nivel de riesgo, el real, el de no modificación de los factores de riesgo y el que controla la variable edad, en conjunto nos permiten valorar de forma más adecuada los beneficios obtenidos con la atención clínica a lo largo del tiempo pues, como ya hemos comentado, sólo podemos intentar amortiguar el riesgo para la salud que es el envejecimiento.

Por otra parte, la opción de analizar la evolución del riesgo relativo, que nos informa de la situación de un individuo con relación a la población de su misma edad y sexo con bajo riesgo, puede ser una alternativa mas útil para valorar la efectividad de las intervenciones realizadas a medio y largo plazo que el riesgo absoluto, pues con el RR estamos controlando la variable edad, que es el principal factor que está influyendo en la modificación del riesgo. Como se ha podido observar hay un descenso constante del RR desde el inicio del estudio, paralelo al descenso de los factores de riesgo cardiovascular y sin embargo la tendencia del riesgo coronario 
absoluto, salvo el descenso inicial, es a un incremento progresivo pese al descenso de los factores de riesgo.

Por lo tanto, podría ser útil monitorizar la evolución del riesgo cardiovascular utilizando el riesgo relativo, una vez planteadas las intervenciones terapéuticas en función del riesgo cardiovascular absoluto, que es el que han utilizado los grandes ensayos clínicos en los que se basan las recomendaciones terapéuticas de las guías clínicas.

La comparación del riesgo cardiovascular estimado con otros estudios no es sencilla pues los resultados de las diferentes escalas no son concordantes habitualmente y con la misma escala pueden variar sustancialmente, en función de la estructura de la muestra (edad y sexo) y prevalencia de determinadas patologías, como pueden mostrar los trabajos de Cristóbal J ${ }^{16}$ (11,36\% en 10 años) y Álvarez $\mathrm{A}^{15}$ (14,9\% en 10 años) .

Resultados similares a los nuestros encuentran Lindholm et $\mathrm{al}^{31}$, que evalúan la efectividad en la reducción de los factores de riesgo principales y del riesgo coronario de una intervención con seis sesiones de educación a los pacientes. Aunque mejoran algunos factores de riesgo, el riesgo coronario global no se modifica. También se comprueba que si los factores de riesgo no se hubiesen modificado el riesgo se elevaría sustancialmente. Finalmente, en el British Family Heart Study ${ }^{32}$ con una intervención para modificar estilos de vida realizada por enfermeras lograron reducir el riesgo coronario en un $16 \%$ en el plazo de un año, siendo estos resultados similares a los nuestros después de una intervención especifica y a corto plazo.

Este estudio podría tener varias limitaciones. En primer lugar el diseño inicial se realizó para un estudio de intervención cuasi-experimental y no para un estudio descriptivo longitudinal. Por otra parte recogimos la información de las historias clínicas de forma ambispectiva y podría haber cierto sesgo en la información. Y en tercer lugar en la muestra hay 58 sujetos $(6,8 \%)$ con cardiopatía isquémica y las tablas están diseñadas para prevención primaria, aunque pensamos que para el tipo de análisis realizado en este trabajo no tiene influencia relevante.

En conclusión, si queremos utilizar la evolución del riesgo coronario para monitorizar la efectividad de la atención sanitaria, debemos tener en cuenta el efecto del paso del tiempo, pues la efectividad clínica lograda quedaría enmascarada por la importante influencia que tiene la edad en la estimación del riesgo cardiovascular. Por esta razón parece adecuado utilizar el riesgo relativo para monitorizar a medio y largo plazo la efectividad de la atención sanitaria.

\section{BIBLIOGRAFÍA}

1. Villar-Álvarez F, Maiques-Galán A, Brotons-Cuixart C, Torcal-Laguna J, Banegas-Banegas JJ, et al. Recomendaciones preventivas cardiovasculares en atención primaria. PAPPS. Aten Primaria 2005;36 Supl 2:11-26.

2. De Backer G, Ambrosioni E, Borch-Johnsen K, Brotons C, Cifkova R, Dallongeville J, et al. Third Joint Task Force of European and Other Societies on Cardiovascular Disease Prevention in Clinical Practice (constituted by representatives of eight societies and by invited experts). European guidelines on cardiovascular disease prevention in clinical practice. Eur J Cardio Prev Rev 2003; 10 (Suppl 1): 1-78.

3. Brotons C, Royo-Bordonada MA, Alvarez-Sala L, Armario P, Artigao R, Conthe P et al. Comité Español Interdisciplinario para la Prevención Cardiovascular (CEIPC). Adaptación española de la Guía Europea de Prevención Cardiovascular. Rev Esp Salud Pública. 2004; 78:435-8.

4. Scottish Intercollegiate Guidelines Network (SIGN). Risk estimation and the prevention of cardiovascular disease. 2007 [Acceso 8 de Julio de 2007] Disponible en http://www.sign.ac.uk/pdf/sign97.pdf.

5. Mann J, Arroll B, Carryer J, et al. New Zealand Guidelines Group (NZGG). The assessment and 
management of cardiovascular risk. Wellington (NZ): New Zealand Guidelines Group (NZGG); 2003 Dec. Disponible en New Zealand Guidelines Group c 1998-2006 [Acceso 8 de julio de 2007];Disponible en: http://www.nzgg.org.nz/guidelines/0035/CVD_Risk_Full.pdf

6. Expert Panel on Detection, Evaluation and Treatment of High Blood Cholesterol in Adults. Executive summary of the third report of the National Cholesterol Education Program (NCEP) (Adult Treatment Panel III). JAMA 2001; 285:2486-97.

7. Mancia G, De Backer G, Dominiczak A, Cifkova R, Fagard R,Germano G et al. 2007 Guidelines for the Management of Arterial Hypertension: The Task Force for the Management of Arterial Hypertension of the European Society of Hypertensión (ESH) and of the European Society of Cardiology (ESC). J Hypertens 2007; 25(6):1105-87.

8. Anderson KM, Wilson PWF, Odell PM, Kannel WB. Un update coronary risk profile, A stament for health professionals. Circulation. 1991;83:356-62.

9. Wilson P; D’Agostino R, Levy D, Belanger A, Silbershatz H, Kannel W. Prediction of Coronary Heart Disease Using Risk Factor Categories. Circulation 1998; 97:1837-47.

10. Grundy SM. Primary prevention of coronary heart disease: integrating risk assessment with intervention. Circulation. 1999 Aug 31;100(9):988-98

11. Marrugat J, Solanas P, D’Agostino R, Sullivan L, Ordovas J, Cordón F, et al. Estimación del riesgo coronario en España mediante la ecuación de Framingham calibrada. Rev Esp Cardiol 2003; 56:253-61.

12. Conroy RM, Pyörala K, Fitzgerald AP, Sans S, Menotti A, De Backer G, et al. on behalf of the SCORE project group. Estimation of ten-year risk of fatal cardiovascular disease in Europe: the SCORE Project. Eur Heart J 2003; 24:987-1003.

13. Stevens RJ, Kothari V, Adler AI, Stratton IM, Holman RR on behalf of the United Kingdom Prospective Diabetes Study (UKPDS) Group.. The UPKDS risk engine: a model for the risk of coronary heart disease in type II diabetes (UKPDS 56). Clin Sci 2001; 101:671-9.

14. Maiques A, Antón F, Taix MF, Albert X, Martí EA, Collado A. Riesgo cardiovascular del SCORE comparado con el de Framingham. Consecuencias del cambio propuesto por las Sociedades Europeas. Med Clin (Barc) 2004; 123:681-5.
15. Alvarez A, Diaz L, Lopez V, Prieto Diaz MA y Suarez S. Comparación de los modelos SCORE y Framingham en el calculo de alto riesgo cardiovascular para muestra de varones de 45 y 65 años de Asturias. Rev Esp Salud Publica 2005; 79:465473.

16. Cristóbal J, Lago F, Fuente J, González-Juanatey JR, Vázquez-Bellés P y Vila M.Ecuación de Framingham de Wilson y ecuación de REGICOR. Estudio comparativo. Rev Esp Cardiol 2005; 58: 910-915.

17. Ramos R, Solanas P, Cordón F, Rohlfs I, Elosua R, Sala J, Masiá R, Faixedas MT y Marrugat J. Comparación de la función de Framingham original y la calibrada del REGICOR en la predicción del riesgo coronario poblacional Med Clin (Barc) 2003; 121:521-6.

18. Baena JM, Val JL, Salas LH, Sanchez R, Altes E, Deixes B, Amatller M y Nuñez D. Comparación de los modelos SCORE y Regidor para el calculo de alto riesgo cardiovascular en sujetos sin enfermedad cardiovascular atendidos en un centro de salud de Barcelona. Rev Esp Salud Publica 2005; 79:453-464.

19. García-Ortiz L, Gómez-Marcos MA, GonzálezElena LJ, Rodríguez-Sánchez E, Garcia Garcia A, Parra-Sanchez J, Herrero Rodriguez C, Gonzalez Garcia A Melón Barrientos L. FraminghamGrundy, Regicor y Score en la estimación del riesgo cardiovascular del paciente hipertenso. Concordancias y discrepancias (CICLO-RISK). Hypertension 2006; 23(3):111-117.

20. Buitrago Ramírez F, Cañón Barroso L Díaz Herrera N, Cruces Muro E, Bravo Simón B y Pérez Sánchez I. Comparación entre la tabla del SCORE y la función Framingham-REGICOR en la estimación del riesgo cardiovascular en una población urbana seguida durante 10 años. Med Clin (Barc) 2006; 127:368-373

21. Buitrago F, Cañón-Barroso L, Díaz-Herrera N, Cruces-Muro E, Escobar-Fernández M y SerranoArias JM. Comparación de las tablas REGICOR y SCORE para la clasificación del riesgo cardiovascular y la identificación de pacientes candidatos a tratamiento hipolipemiante o antihipertensivo. Rev Esp Cardiol. 2007; 60:139-47.

22. Bowman TS, Sesso HD, Gaziano JM. Effect of age on blood pressure parameters and risk of cardiovascular death in men. Am J Hypertens 2006;19(1):47-52.

23. Wang W, Lee ET, Fabsitz RR, Devereux R, Best L, Welty TK, Howard BV. A longitudinal study of 
hypertension risk factors and their relation to cardiovascular disease: the Strong Heart Study. Hypertension 2006; 47(3):403-9.

24. Coca A, Dalfó A, Esmatjes E, Llisterri JL, Ordoñez J, Gomis R, Gonzalez-Juanatey JR, MartinZurro A; Grupo PREVENCAT. Treatment and control of cardiovascular risk in primary care in Spain. The PREVENCAT study Med Clin (Barc) 2006; 126(6):201-5.

25. Baena-Díez JM, García-Lareo M, de la PozaAbad M, Hernandez-Ibanez R, Munoz-Rubio A, García-Rey Z. Estimation of overall cardiovascular risk from coronary risk. A cohort study. Med Clin (Barc) 2006; 127(1):8-10.

26. Grundy SM, Pasternak R, Greenland P, Smith S Jr, Fuster V. Assessment of cardiovascular risk by use of multiple-risk-factor assessment equations: a statement for healthcare professionals from the American Heart Association and the American College of Cardiology. Circulation 1999; 100(13):1481-92.

27. García Ortiz L, Santos Rodríguez I, Sánchez Fernández PL, Mora Santiago MC Arganda Maya J, Rodríguez Corral T. Efectividad de una intervención de mejora de calidad en la reducción del riesgo cardiovascular en pacientes hipertensos. Revista Española de Cardiología 2004; 57: 664-51.
28. Gómez Marcos MA, García Ortiz L, Sánchez Rodríguez A, Melón Barrientos L, Herrero Rodriguez C, Bodego Sánchez P. Mejora de calidad del proceso asistencial a los factores de riesgo cardiovascular en el paciente hipertenso. eficacia de una intervención ( CICLO-RISK). Hipertensión 2005; 22:345-52.

29. Gómez Marcos Manuel A,. García Ortiz Luis,. Sánchez Rodríguez Á, González Elena LJ. Efectividad de una intervención de mejora de calidad en la reducción del riesgo coronario y del riesgo de mortalidad cardiovascular en pacientes hipertensos. Atención Primaria 2006; 37:498-503.

30. Gómez Marcos MA, García Ortiz L, González Elena LJ, Ramos Delgado E, González García A, Parra Sánchez J. Efectividad de una intervención de mejora de calidad en el control de la presión arterial en atención primaria. Revista Clínica Española 2006; 206 (9):428-34 .

31. Lindholm LH, Ekbom T, Dash C, Eriksson M, Tibblin G, Schersten B, The impact of health care advice given in primary care on cardiovascular risk. BMJ 1995; 310: 1105-9.

32. Family Heart Study Group. Randomised controlled trial evaluating cardiovascular screening and intervention in general practice: principal results of British Family heart Study. BMJ 1994; 308:313-320. 